\title{
Études de gradients de dissémination dans le cas de l'oïdium du haricot en Guadeloupe
}

\author{
P Pauvert \\ INRA, station de pathologie végétale, de phytoécologie et de malherbologie, BP 1232, 97184, Pointe-à-Pitre Cedex, France
}

(Reçu le 25 septembre 1990; accepté le 4 décembre 1990)

\begin{abstract}
Résumé - En Guadeloupe, la régularité des alizés (secteur est) permet d'étudier des gradients de dispersion de maladies disséminées par le vent. La progression de l'oïdium (Erysiphe polygoni DC) a été notée dans 6 variétés de haricot (Phaseolus vulgaris L) placées à des distances variables d'une source d'inoculum constituée par une parcelle infectée de la variété sensible Borlotto nano vigevano située dans la partie est de l'essai. La transformation de Gregory (1968) traduit correctement la progression moyenne de l'oïdium. La progression dans chaque variété est influencée par la résistance à la maladie, celle-ci s'exprimant parfois à l'état adulte. Il apparaît que quelles que soient les variétés, le rendement est diminué au voisinage de la source d'inoculum, les mécanismes de résistance consommant de l'énergie. En pratique, des variétés résistantes placées près de parcelles infectées peuvent subir des pertes de rendement non décelées.
\end{abstract}

haricot / oïdium / gradient de dispersion / perte de rendement / résistance

Summary - Studies on bean powdery mildew in Guadeloupe. In Guadeloupe trade winds of regular direction provide good conditions for epidemiological studies on wind-borne disease gradients. Six varieties of bean (Phaseolus vulgaris $L$ ) were studied for powdery mildew progression (Erysiphe polygoni $D C$ ). The source of inoculum came from a plot of the susceptible variety Borlotto nano vigevano in the eastern part of the field (fig 1). The resulting powdery mildew gradient was measured on 4 different dates (fig 2). Regression analysis of mean disease severity with distance indicated that the log-log disease spread model fitted the data (table I). The apparent variety susceptibility estimated by the area under the severity curve (table II) affected the powdery mildew spread. However, resistance mechanisms resulted in an energy expenditure, and all the varieties showed a yield decrease near the source of inoculum (figs 3, 4). So in practice, the vicinity of infected crops may reduce the performance of bean resistant varieties which are apparently symptom-free.

bean / powdery mildew / dispersal gradient / yield loss / resistance

\section{INTRODUCTION}

Aux Antilles, l'oïdium (Erysiphe polygoni DC) est une importante maladie foliaire du haricot ( $P$ haseolus vulgaris $L$ ) pendant la saison sèche (décembre-mai). Le parasite survit pendant la saison humide sur les légumineuses sauvages, souvent sous des arbres qui lui procurent :

- une protection des conidiophores contre les fortes pluies tropicales;

- un ombrage favorable à son développement.

Ce dernier point a été vérifié récemment à la suite du passage de l'ouragan Hugo (16-17 septembre 1989), l'ébranchage et la défoliation des arbres ont entraîné une diminution sensible des attaques d'oïdium dans les mois qui ont suivi.
Des études récentes ont montré l'existence en Guadeloupe de 2 races physiologiques d'oïdium (Pauvert, 1989). La race 2 prédomine en fin de saison sèche et affecte un plus grand nombre de variétés que la race 1 .

L'existence aux Antilles d'une grande régularité de la direction des vents (alizés d'est-sud-est) est un avantage important pour étudier des maladies à dissémination aérienne. La possibilité de créer des gradients de maladie à partir d'un foyer artificiel d'inoculum primaire est intéressante pour étudier la progression d'une épidémie, et aussi pour tenter d'établir le lien existant entre l'importance des attaques et les baisses de rendement consécutives, domaine où les informations sont peu nombreuses, en particulier pour l'oïdium du haricot; Schwartz et al (1981). 


\section{MATÉRIEL ET MÉTHODES}

\section{Matériel végétal}

Les semis sont effectués, sur un terrain rectangulaire constitué par un sol alluvial particulièrement homogène, selon des rangs orientés est-ouest, c'est-à-dire dans le sens du vent dominant. Les parcelles élémentaires sont constituées par des lignes de $3 \mathrm{~m}$ de longueur (56 grains semés) disposées de façon que l'on retrouve pour chacune des 6 variétés utilisées 6 distances progressives par rapport à la source d'inoculum constituée par une bordure de la variété italienne très sensible Borlotto nano vigevano, située dans le prolongement de l'essai et à l'est. Les variétés utilisées, sélectionnées par Messiaen et al (1989) ont été :

- Nicanor 2-4 : lignée pure, grain noir, croissance indéterminée, tolérance aux 2 races d'oïdium;

- Mersant : population, grain noir, croissance indéterminée, tolérance aux 2 races d'oïdium;

- 59-11-8 : lignée pure, grain noir, croissance indéterminée, résistance monogénique à la race 1 d'oïdium, tolérance à la race 2;

- CNR1 : lignée pure, grain rouge foncé, rayé, croissance déterminée, résistance monogénique à la race 1 d'oïdium, faible tolérance à la race 2;

- CNR44 : lignée pure, grain rouge uni, croissance déterminée, résistance monogénique à la race 1 d'oïdium, sensibilité à la race 2;

- Salagnac 90 : lignée pure, grain panaché rouge/ rose, tolérance aux 2 races d'oïdium.

\section{Sources d'inoculum}

L'inoculum primaire provient de feuilles de Pueraria phaseoloides, plante sauvage sur laquelle survit l'oïdium pendant la saison chaude et humide (Messiaen et al, 1989). Les feuilles sont secouées au-dessus de plantules en serre de la variété italienne Borlotto vigevano, particulièrement sensible à l'oïdium. Cette variété semée hebdomadairement permet de disposer d'un réservoir d'inoculum permanent. Les contaminations d'une série à l'autre se font naturellement sous l'influence du vent (les serres sont largement ouvertes) et peuvent être aussi favorisées en soufflant quotidiennement sur les plantes très infectées au voisinage de la série suivante.

La variété Borlotto vigevano héberge en novembredécembre la race 1, puis un mélange de la race 1 et de la race 2 par la suite. Les plantules apportées au champ comportent un mélange des 2 races en proportion variable.

\section{Notation de la maladie}

La sévérité moyenne des attaques d'oïdium par parcelle élémentaire (proportion de feuillage malade) est estimée dès l'apparition des pustules, tous les $7 \mathrm{j}$. Une notation plus fine, (plante par plante) est effectuée dans la parcelle contaminatrice (variété Borlotto), dans les premiers stades de l'infection.

A la récolte, on détermine, pour les 6 variétés, le poids de grains secs par parcelle, ainsi que le poids moyen d'un grain.

\section{RÉSULTATS}

\section{Progression de la maladie dans la variété contaminatrice Borlotto}

Cette progression a été étudiée plus particulièrement en 1987. Le semis a été effectué le 27 février et les pots contenant des plants de Borlotto très infectés ont été apportés le 11 mars. Dès le 18 , des pustules situées sur les plantes les plus proches des pots contaminateurs sont observées, conformément aux résultats d'expériences postérieures à cet essai, montrant que la période de latence est d'environ $3 \mathrm{j}$ à $23^{\circ} \mathrm{C}$ et augmente au-dessus de cette température (Pauvert, 1989). La notation du 23 mars (fig 1) met en évidence l'effet déterminant de la régularité en direction de l'alizé, qui se traduit par un gradient très net de sévérité d'oïdium «sous le vent" des plantes en

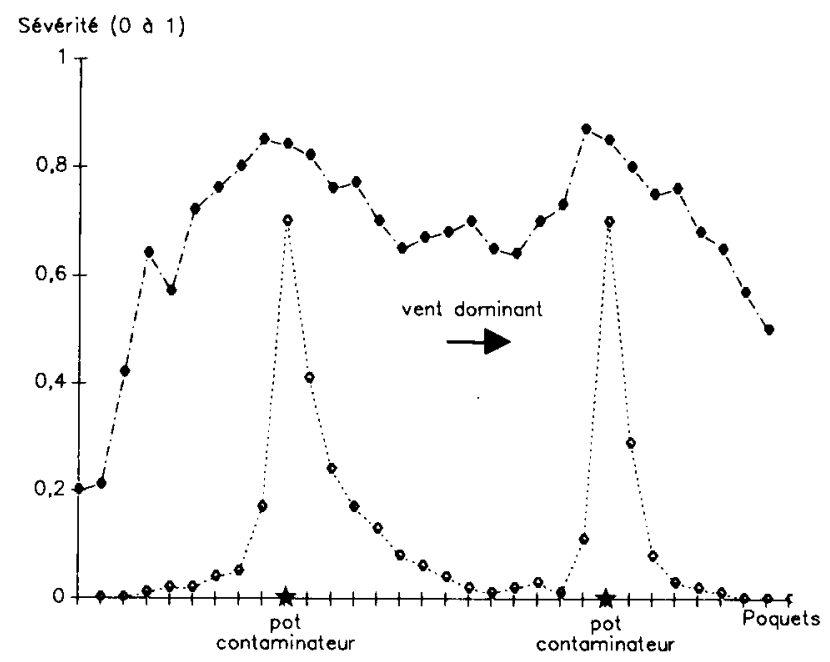

Fig 1. Progression de l'oïdium de poquet à poquet dans la parcelle servant de source d'inoculum (var Borlotto) à partir de 2 pots contaminateurs par rang: sévérité par poquet le $-0-23$ mars et le $-^{\circ}-30$ mars. 
pot contaminatrices. Le 30 mars, l'effet du vent dominant est toujours perceptible mais le gradient tend à s'estomper. On peut donc considérer qu'à cette date ure source relativement homogène d'inoculum existait pour les autres variétés.

\section{Progression de la maladie dans les variétés testées}

\section{Progression moyenne}

La progression moyenne de la maladie au sein des 6 variétés est représentée sur la figure 2 . Le gradient de maladie est très net le 30 mars et le 6 avril. En revanche, à partir du 13 et surtout le 20 avril, la sévérité moyenne est à peu près la même, quelle que soit la distance au foyer initial. Les caractéristiques de ce gradient peuvent être quantifiées en opérant la transformation de Gregory (1968) :

$$
\log (s)=a+b \cdot \log (d)
$$

où $s$ est la sévérité de la maladie, $d$ la distance au foyer en $\mathrm{m}$ (tableau I).

Le coefficient $b$, (pente de la droite de régression) mesure la vitesse de diminution de la maladie en fonction de la distance. Les valeurs trouvées sont conformes à l'étude de Gregory (1968). Progressivement $b$ se rapproche de la valeur 0 , ce qui est la conséquence de contaminations secondaires. Cette extension rapide de la maladie dans l'espace est la conséquence de la faible durée de la période de latence, de l'intense sporulation du parasite et de son mode de dissémination.

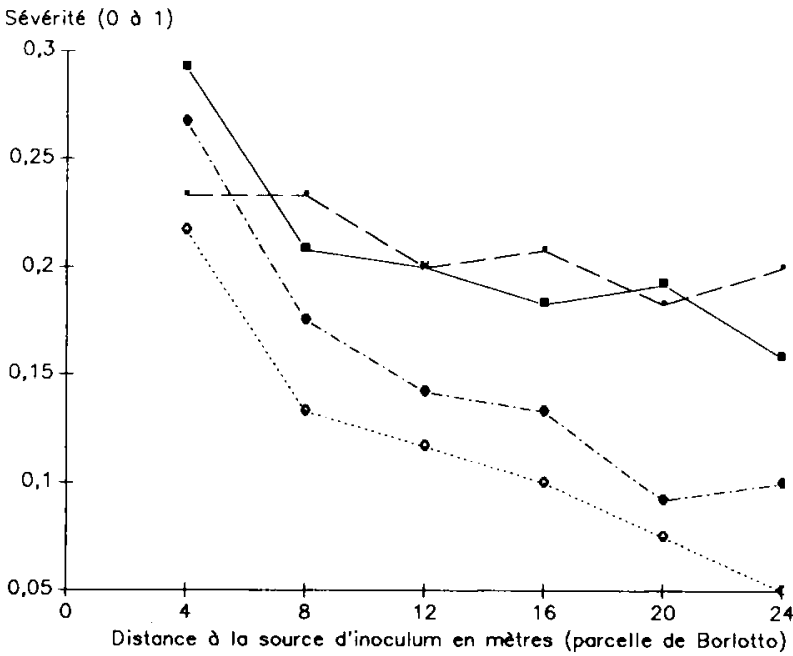

Fig 2. Gradient moyen d'oïdium à 4 dates : -O- 30 mars; 6 avril; - -13 avril; -- -20 avril.

Tableau I. Coefficients $a$ et $b$ (transformation de Gregory). $r$ : coefficient de corrélation.

$$
30 \text { mars } 6 \text { avril } 13 \text { avril } 20 \text { avril }
$$

$\begin{array}{lllll}b & -0,726 & -0,575 & -0,251 & -0,120 \\ a & -0,462 & -0,527 & -0,877 & -1,267 \\ r & -0,958 & -0,977 & -0,948 & -0,837\end{array}$

\section{Variétés indéterminées}

Sur ces variétés, la sévérité moyenne est faible au 30 mars $(0,12)$, puis devient nulle. L'oïdium ne s'exprime que pendant une période très limitée et à proximité immédiate de la source d'inoculum. Dans les conditions habituelles de culture ces variétés ne sont pas ou très peu attaquées.

\section{Variétés déterminées}

Sur la variété $S 90$, l'oïdium augmente progressivement jusqu'au 13 avril, puis régresse. Sur les variétés CNR1 et CNR44, l'oïdium progresse de façon régulière, et ces 2 variétés ont pratiquement le même comportement.

Globalement la quantité moyenne de maladie ayant affecté les variétés peut être matérialisée par l'aire sous la courbe de sévérité (tableau II).

\section{Effet du gradient sur le rendement}

Dans les limites du terrain de l'essai, le rendement augmente au fur et à mesure que l'on s'éloigne de la source d'inoculum (fig 3). L'inexistence d'un gradient de fertilité qui aurait

Tableau II. Aire sous la courbe de sévérité (sévérité $\mathrm{x}$ jours).

\begin{tabular}{llll}
\multicolumn{2}{l}{ Variétés indéterminées } & \multicolumn{2}{c}{ Variétés déterminées } \\
variétés & aire & variétés & aire \\
& & & \\
\hline 59-11-8 & 0,056 & S90 & 3,47 \\
Mersant & 1,008 & CNR1 & 9,94 \\
Nic 2-4 & 1,169 & CNR44 & 8,34
\end{tabular}




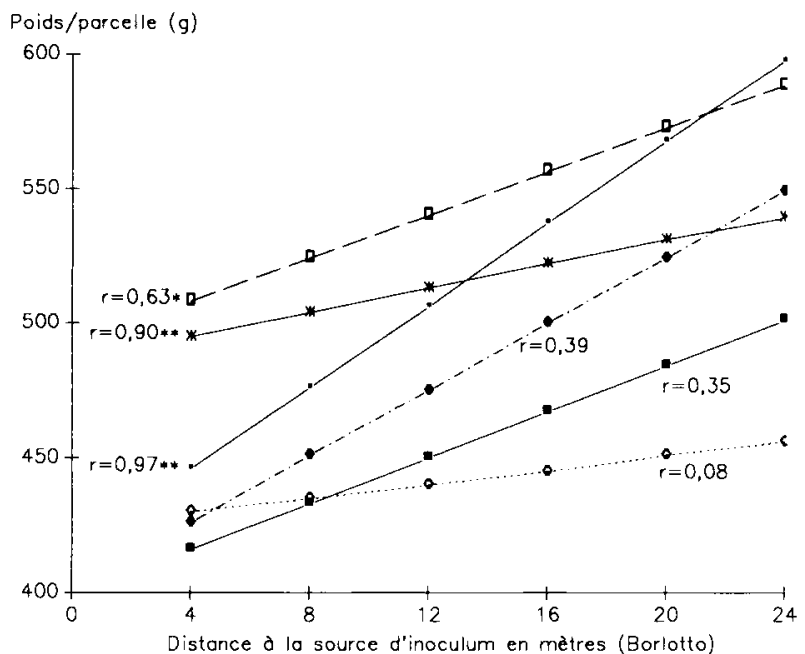

Fig 3. Rendement des variétés en fonction de la distance à la source d'inoculum (droites de régression). -O- CNR1; - -

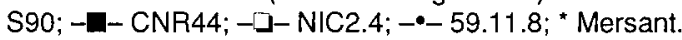

faussé les résultats est démontrée par la différence de comportement entre variétés sensibles et résistantes. Pour les variétés sensibles sur lesquelles la maladie s'est nettement propagée selon un gradient, l'accroissement de rendement en fonction de la distance est faible, ce qui est la conséquence des contaminations secondaires. Sur les variétés indéterminées résistantes, pour lesquelles on pourrait s'attendre à ce que la maladie n'ait aucun effet sur le rendement on assiste, au contraire à une nette diminution près du foyer d'inoculum, effet le plus marqué sur la variété 59.11 .8 , pourvue du plus haut niveau de résistance aux 2 races.

Cette perte de rendement est surtout la conséquence de la diminution du poids moyen des grains (fig 4).

\section{CONCLUSION}

La réduction du rendement de plantes sous l'influence du dépôt de spores avirulentes est un phénomène déjà mis en évidence (SmedegaardPetersen, 1981), en particulier chez l'oïdium de l'orge (Erysiphe graminis $\mathrm{f} s \mathrm{sp}$ hordel). La réaction de défense induite par le pathogène utilise de l'énergie et se traduit par une augmentation de la consommation d'oxygène $6-9 \mathrm{~h}$ après l'inoculation; ce qui correspond aux premières tentatives de pénétration à partir de l'appressorium.

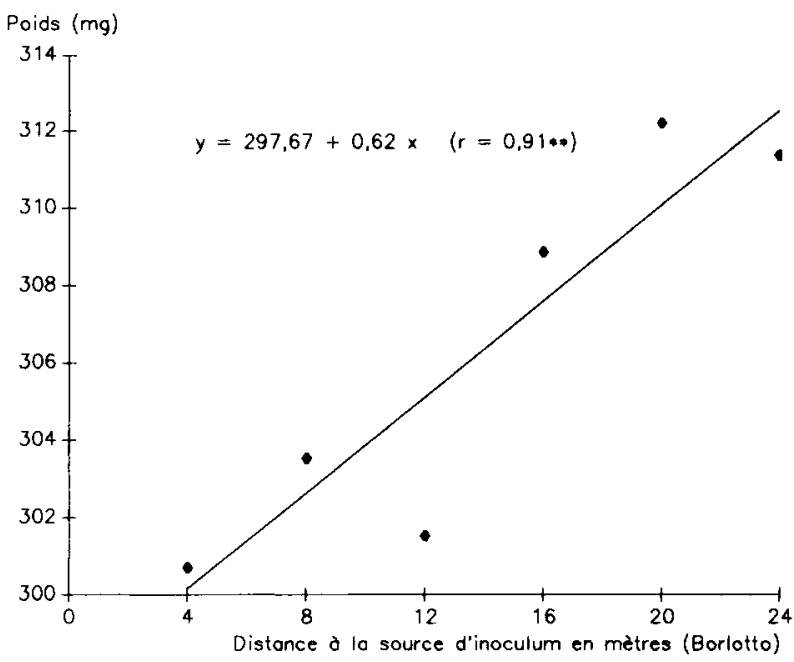

Fig 4. Poids moyen des grains en fonction de la distance à la source d'inoculum (toutes variétés).

De plus, alors que cette consommation d'oxygène revient à la normale au bout de $3 \mathrm{j}$ chez une plante non soumise à de nouvelles contaminations, chez une plante soumise à des contaminations successives, l'augmentation de respiration reste permanente. Nos résultats semblent indiquer qu'il en est probablement de même dans le cas de l'oïdium du haricot.

Sur le plan pratique, il en résulte que des parcelles de cultures apparemment saines peuvent subir des pertes de rendement tout à fait occultes si elles sont placées sous le vent de plantes plus âgées infectées. Sur le plan de la sélection vis-à-vis de la résistance à l'oïdium, la mesure de l'importance de la perturbation de la respiration pourrait apporter un utile complément d'information pour la notation des variétés.

\section{REMERCIEMENTS}

L'auteur remercie CM Messiaen pour la fourniture du matériel végétal et l'aide de ses conseils, $C$ Vincent et l'équipe de la station d'amélioration des plantes INRA Antilles-Guyane pour la mise en place, l'entretien et l'égrenage des essais.

\section{RÉFÉRENCES}

Gregory PH (1968) Interpreting plant disease dispersal gradients. Annu Rev Phytopathol 6, 189-212 
Messiaen CM, Pauvert P, Jacqua G, Laraque A (1989) L'oïdium américain du Haricot (Erysiphe polygoni $\mathrm{DC})$ dans la zone antillaise; recherche de géniteurs de résistance. Agronomie 9, 259-263

Pauvert P (1989) Contribution à l'étude des races d'oïdium du haricot (Erysiphe polygoni DC) en Guadeloupe. Agronomie 9, 265-269
Schwartz HF, Katherman MJ, Thung MTD (1981) Yield response and resistance of beans to powdery mildew in Colombia. Plant Dis 65, 737-738

Smedegaard-Petersen V, Stølen O (1981) Effect of energy-requiring defense reactions on yield and grain quality in a powdery mildew resistant barley cultivar. Phytopathology 71, 396-399 